\title{
IDENTIFICATION OF EXCITATORY AND INHIBITORY MOTONEURONS IN THE NEMATODE ASCARIS BY ELECTROPHYSIOLOGICAL TECHNIQUES ${ }^{1}$
}

\author{
JOHN P. WALROND, ${ }^{2}$ IRA S. KASS, ANTONY O. W. STRETTON, ${ }^{3}$ and JUDITH E. DONMOYER \\ Department of Zoology, University of Wisconsin, Madison, Wisconsin 53706
}

Received May 28, 1981; Revised July 23, 1984; Accepted July 25, 1984

\begin{abstract}
A physiological preparation in which it is possible to record responses in muscle to stimulation of single motoneurons of the nematode Ascaris lumbricoides is described. With this preparation we have determined the physiological sign (E or I; excitatory or inhibitory) of the neuromuscular synapses of 21 identified motoneurons -12 are excitatory and 9 inhibitory. Ascaris motoncurons had previously been classified by morphological criteria into seven classes (Stretton, A. O. W., R. M. Fishpool, E. Southgate, J. E. Donmoyer, J. P. Walrond, J. E. R. Moses, and I. S. Kass (1978) Proc. Natl. Acad. Sci. U. S. A. 75: 3493-3497). Physiological studies were performed on members of five of these classes. Three classes of neurons (DE1, DE2, and DE3) are excitatory to dorsal muscle cells. Two classes (DI and VI) are inhibitory neurons which innervate the dorsal and ventral muscle cells, respectively. The motoneurons in Caenorhabditis elegans (White, J. E., E. Southgate, J. N. Thomson, and S. Brenner (1976) Philos. Trans. R. Soc. Lond. (Biol.) 275: 327-348) can be divided into seven morphological classes which are very similar to those in Ascaris. Based upon the structure-function correlation in Ascaris, we have predicted which motoneurons are excitatory and which are inhibitory in C. elegans.
\end{abstract}

A basic tenet of modern neurobiology is that behavior is under the control of the nervous system; behavioral coordination is thought to emerge from the physiological properties of the individual neurons and their synaptic connections. As early as 1908, Richard Goldschmidt proposed that the large nematode Ascaris would provide an ideal system in which to investigate the neural control of behavior since this animal contains only a small number $(\sim 250)$ of nerve cells. In a classic series of experiments, Goldschmidt (1908, 1909) reconstructed the anatomy of the neurons in the head (Goldschmidt, 1908) and tail (Goldschmidt, 1909) showing that they were completely reproducible from animal to animal and that all of the neurons could be individually recognized and named. Having made these important observations, Goldschmidt (1908, 1909) realized that, without information about the physiological properties of

\footnotetext{
${ }^{1}$ We thank A. Chambers, A. Aaroen, C. Hughes, and D. Chandler for their help in preparing the manuscript. We also thank Dr. C. D. Johnson for the benefit of many stimulating discussions, and both Dr. Johnson and Dr. C. Kung for critical reading of the manuscript. This work was supported by United States Public Health Service Grants NS 10509 and AI 15429, National Science Foundation Grant. RNS 7609641, a Sloan Fellowship to A. O. W. S., a research grant from the Muscular Dystrophy Association of America, and the Research Fund of the Graduate School, University of Wisconsin-Madison. I. S. K. was supported by United States Public Health Service Training Grant GM 07215.

${ }^{2}$ Present address: Laboratory of Neurobiology, National Institute of Neurological and Communicative Disorders and Stroke, Marine Biological Laboratory, Woods Hole, MA 02543.

${ }^{3}$ To whom correspondence should be addressed.
}

the cells, little additional progress could be made; therefore, he abandoned the project.

Since Goldschmidt's time electrophysiological techniques have become available to investigate the role of nerve cells in nematode behavior. Nematode muscle cells are segregated into two fields, dorsal and ventral, which are innervated by motoneuron processes in the dorsal and ventral nerve cords, respectively. Intracellular electrical recordings from nematode muscle cells were first reported by Jarman (1959), who demonstrated the existence of complex spontaneous activity in muscle cells of Ascaris lumbricoides. Subsequently, it was shown that stimulating the nervous system could evoke either excitatory or inhibitory responses in muscle cells (de Bell et al., 1963; del Castillo et al., 1967; Weisblat and Russell, 1976), suggesting the existence of both excitatory and inhibitory motoneurons. However, because these investigators lacked a detailed knowledge of the anatomy of the motor nervous system of Ascaris they could not relate these responses to individual motoneurons.

To identify individual motoneurons for physiological experiments, we have recently extended the classical analysis of Ascaris neuroanatomy to the ventral and dorsal nerve cords which contain the cell bodies and processes of the motoneurons which innervate the muscle cells (Stretton et al., 1978). Each motoneuron innervates either dorsal or ventral muscle cells. Based upon their characteristic shapes, the motoneurons have been divided into seven distinct classes, each of which is present in several copies. Members of all seven classes have a cell body and a process in the ventral nerve cord. Five types also have a dorsal process linked to the ventral process by a single transverse fiber. These single fibers, first described by Hesse (1892), 
have been called commissures; ${ }^{4}$ they are arranged in a highly reproducible pattern in the body of the worm.

Because the position of the commissures is so reproducible, we can make dissections in which the commissure of a single identified motoneuron is the only neuronal connection between the dorsal and ventral nerve cords. In these preparations, we determined the sign of the neuromuscular output of the motoneuron by stimulating it in one nerve cord while recording from the muscle cells it innervates in the other cord.

Of the five neuronal types with commissures, four are dorsal motoneurons and one is a ventral motoneuron. The present results show that members of three classes of motoneurons (DE1, DE2, and DE3) are excitatory to dorsal muscle cells, one class (DI) is inhibitory to dorsal muscle cells, and one class (VI) is inhibitory to ventral muscle cells. (Preliminary reports of these findings have appeared elsewhere (Walrond et al., 1977; Stretton et al., 1978).)

Attaining an understanding of how Ascaris uses its nervous system to control behavior will requirc more than a knowledge of the anatomy and neuromuscular physiology of individual motoneurons. It will also require insights into how the motoneurons are controlled to produce coordinated output. The following paper (Walrond and Stretton, 1985a) presents evidence for synaptic interactions between the dorsal excitatory and ventral inhibitory motoneurons. The third paper (Walrond and Stretton, 1985b) presents physiological evidence for synaptic interactions among the dorsal motoneurons and discusses how these neuron-neuron interactions lead to plausible models of how the nematode nervous system controls locomotion. These models are currently being tested and refined by intracellular recording from Ascaris neurons (Davis and Stretton, $1981,1982,1983)$.

\section{Materials and Methods}

Worms were obtained from the intestines of freshly killed hogs at a local slaughterhouse and transported to the laboratory in Kronecker's solution $(0.9 \% \mathrm{NaCl}$ and $0.15 \mathrm{~mm} \mathrm{NaOH})$. In the laboratory, animals were placed in fresh Kronecker's solution and maintained at $37^{\circ} \mathrm{C}$ for up to 4 days. The Ascaris saline solution used in electrophysiological experiments contains $24.5 \mathrm{mM} \mathrm{KCl}, 11.8 \mathrm{mM} \mathrm{CaCl}_{2}, 9.8 \mathrm{mM} \mathrm{MgCl}_{2}, 7.8$ $\mathrm{mM} \mathrm{NaCl}, 125 \mathrm{mM}$ sodium acetate buffered with Tris or TES $(\mathrm{N}$-Tris(hydroxymethyl)methyl-2-aminoethane sulfonic acid), $\mathrm{pH}$ 7.4. This solution, which has twice the levels of $\mathrm{Ca}^{2+}$ and $\mathrm{Mg}^{2+}$ present in Ascaris body fluids, suppresses the spontaneous activity of muscle cells so that individual responses to stimulation of single motoneurons can be more easily observed.

In intact animals, the commissures can be visually identified in a dissecting microscope under darkfield illumination. 'The commissures are arranged in a repeating pattern (Fig. $1 A$ ); each unit or segment contains seven commissures (Fig. $1 B$ ). Typically, there are three pairs that traverse the right side of the animal and a single left-hand commissure (Fig. 1C). The seven commissures represent five types of motoneurons (Fig. $1 D$ ). The first and second pairs of commissures to the right in a segment each consist of a DE1 and a VI motoneuron. DE1 and VI commissures can be distinguished because the diameter of the DE1 commissure is greater than that of the VI; DE1 is also usually anterior to VI (C. D. Johnson and A. O. W. Stretton, submitted for publication). The members of the third right-hand pair, commissures of DE2 and DI motoneurons, often cannot be distinguished by their diameters, but since the DE2 commissure lies anterior to the DI neuron about $70 \%$ of the time (C. D. Johnson and A. O. W. Stretton, submitted for publication), they can usually be distinguished by their relative position. The commissures of DE3 motoneurons are easily identified since they are the single left-hand commissures in each segment.

${ }^{4}$ It should be noted that this is a special use of the term "commissure" that is established in the nematode literature (see Bullock and Horridge, 1965). In contrast to other systems in which commissures are bundles of fibers linking left-right symmetrical structures, in nematodes the term refers to single dorsoventral fibers.
To study reponses to stimulation of motoneurons, preparations in which the ventral and dorsal nerve cords remained connected by single commissures were made. For commissures in right-hand pairs, one member of the pair was severed by puncturing the body wall and commissure with an insect pin. A 4-cm section of worm containing the unsevered commissure was then excised. The left lateral line was cut completely and the right lateral line was cut partially so as to leave the dorsal and ventral halves connected by a narrow strip of body wall containing a single uncut neuron (Fig. 2). For DE3, complementary dissections were carried out, leaving a tissue bridge containing the single commissure which crosses the left lateral line, while completely severing the right lateral line. The preparation was then pinned out in the physiological recording chamber (volume, $20 \mathrm{ml}$ ) with its muscle surface uppermost, and the intestine was removed with forceps. The bath was maintained at 37 to $38^{\circ} \mathrm{C}$ with an electrical heating coil and fresh Ascaris saline, preheated in a reservoir, was perfused through the chamber at $90 \mathrm{ml} / \mathrm{hr}$. A narrow longitudinal strip of body wall containing a length of nerve cord that was known from anatomical data (Stretton et al., 1978) to contain a process of the intact neuron was dissected and drawn up into a suction electrode for stimulation with either single square current pulses or trains of pulses.

Intracellular recordings were made from muscle cell bellies (Fig. 3). In nematodes, muscle cells have three distinct parts: the spindle, containing the contractile fibers; the belly, containing the nucleus; and the arm, which extends from the belly to the nerve cord where it branches and receives synapses from the motoneurons (Rosenbluth, 1965). Penetrations were made with $\mathrm{KCl}$ - or $\mathrm{KOAc}$-filled microelectrodes (20 to 40 megohms) mounted on free-floating chloridized silver wire and connected through a high impedance preamplifier to an oscilloscope. Muscle cell resting potentials were generally 35 to $40 \mathrm{mV}$. In all cases, muscle cell bellies close to the nerve cord were chosen for recording evoked responses since, as previously reported by del Castillo et al. (1967), the amplitude of muscle responses decreased with increasing displacement from the nerve cord (see also Walrond, 1979). When the input (dendritic) process of the intact cell was stimulated in one nerve cord, intracellular recordings were taken from muscle cells known anatomically to be directly innervated by the neuron (Stretton et al., 1978 ) in the other nerve cord. When the output region of a neuron was stimulated, intracellular recordings were taken from muscle cells within the neuron's input region. In these latter experiments, a signal averager (Tracor Northern 570) was used to increase the probability of detecting low amplitude responses.

To show that the observed response resulted from activation of the motoneuron, and not from direct electrical activation of the muscle cells by current spread from the stimulating electrode, in many experiments the intact commissure was cut while monitoring the evoked activity. Because commissures can be seen in intact animals but not after dissection, the position of a commissure pair was marked by puncturing the body wall of the intact animal with an insect pin coated with carmine particles. This technique leaves a red dot inside the body wall. Two such marks were used to span the position of a selected commissure. By cutting only the short section of the body wall between the marks, the intact commissure could be severed without unduly disturbing the microelectrode recording the evoked response.

Following the electrophysiological analysis, many preparations were fixed, embedded, and serially sectioned for light microscopy (Stretton, 1976). In order to validate the surgery used to isolate commissures and to identify the commissural neurons, the profiles of the cut and intact fibers were traced through serial sections, and each member of the pair was identified by the position of its cell body and the course taken by its processes in the dorsal and ventral cords (Stretton et al., 1978).

\section{Results}

Each of the first and second pairs of commissures in each repeating unit includes the commissure of a DE1 neuron (Fig. 1). Activation of the ventral process of either of these motoneurons produced a twitch in the dorsal musculature. Intracellular recordings taken from dorsal muscle cells in the region where the stimulated DE1 motoneuron has neuromuscular synapses revealed a slow depolarizing response or a slow depolarization with a superimposed spike (Fig. $3 A$ ). These spikes occasionally reached an amplitude of 30 to $40 \mathrm{mV}$ but only rarely overshot the $0 \mathrm{mV}$ potential. Spikes were only secn in muscle cells close to the nerve cord; the response evoked in 


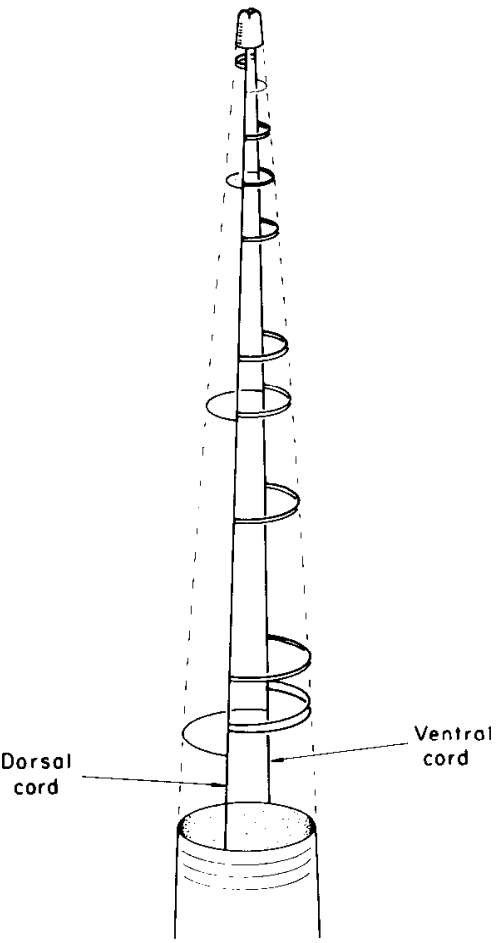

A

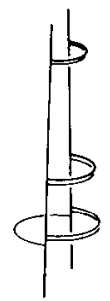

B.

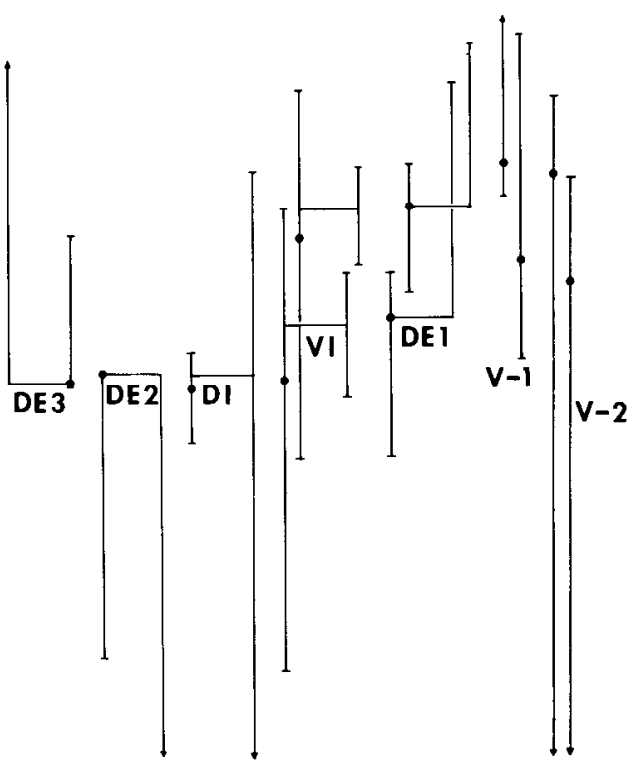

C

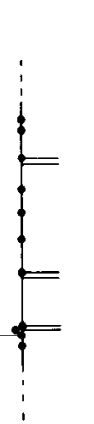

Figure 1. Diagrammatic representation of the motoneurons of Ascaris. A, Diagram showing the dorsal and ventral nerve cords connected by the commissures. Commissures occur in a repeating pattern of three pairs to the right followed by a single to the left. The diagram shows the first three (of five total) repeating units. The number and arrangenent of commissures in the second $(B)$ and third repeats are identical; the first segment contains an extra right-hand commissure, and the most anterior pair is to the left. $C$, Diagram of the ventral nerve cord showing the position of motoneuron cell bodies, and commissures to the right and to the left in a typical second segment. $D$, Diagram showing the dorsal and ventral processes of the second segment motoneurons. The vertical lines represent the processes within the nerve cords, and the horizontal lines represent the commissures. The ventral processes may be recognized since they contain the cell body (black dots). The bars at the ends of the vertical lines show where the processes terminate. The arrowheads indicate that the processes extend farther along the cord. The neurons are in their correct relative position longitudinally but have been spread laterally to facilitate viewing. Of the commissural motoneurons, each repeat contains one copy of neuron types DE3, DE2, and DI, and two copies of neuron types VI and DE1; in addition, each repeat includes two copies each of neuronal types V-1 and V-2.

more lateral muscle cells consisted of the slow potential alone. All six of the DE 1 neurons in the first three segments evoked depolarizing responses in the dorsal musculature. We therefore concluded that the DF1 neurons are excitatory motoneurons.

The neurons designated DE2 and DE3 occur in only one copy per segment. In each segment the commissure of the DE2 neuron is a member of the third pair of commissures, and DE3 is the neuron with the single left-hand commissure (Fig. 1). Stimulating the ventral process of either of these neurons evoked twitches and depolarizing responses in the dorsal musculature (Fig. 3, $B$ and $C$ ) similar to responses evoked by activation of the DE1 motoneurons. Each of the three DE2 neurons and the three DE3 neurons of the first three segments showed similar responses. These results indicate that the DE2 and DE3 neurons are also excitatory motoneurons.

The other neuron present in the third pair of commissures is a type DI neuron (Fig. 1). Activation of the ventral process of this neuron evoked hyperpolarizing responses in the dorsal musculature (Fig. 4A). Each DI neuron in the first three segments evoked similar responses; in addition, stimulating a DI neuron in preparations which showed spontaneous excitatory activity in the dorsal muscles reduced or eliminated the depolarizing potentials. Therefore, we have concluded that the DI neuron is a dorsal inhibitory motoneuron.

The other member of the first and second pair of commissures is a VI motoneuron process (Fig. 2). The VI neurons synapse with the ventral musculature (Siretlon et al., 1978). Activating the dorsal process of a VI neuron produces a hyperpolarization in the ventral musculature (Fig. $4 B$ ). These hyperpolarizations are indistinguishable from the ones evoked in dorsal muscles by the ventral activation of the DI neuron. All six of the VI neurons in the first three segments evoke similar ventral hyperpolarizations. Thus, these neurons are ventral inhibitory motoneurons.

In some experiments, stimulation of single excitatory or inhibitory motoneurons produced complex responses. For DE neurons a hyperpolarizing potential often followed the depolarization (Fig. 5A), and for the inhibitory neurons a depolarizing potential often followed the evoked hyperpolarization (Fig. $5 B$ ). In different muscle cells, the relative amplitude of the first and second components of the response varied. Although the present experiments do not define the origin of these responses, they may result from synaptic interactions within the nerve cords or from electrical properties of nerve or muscle cells (see "Discussion").

When the commissure was cut while recording the evoked response, the response was obliterated (Fig. 6, Table I). These results showed that the evoked potential resulted from activity conducted through the commissural neuron and not from the direct electrical activation of muscle cells by current leaking out of the stimulating electrode.

Stimulating the DE neurons with varying extracellular current pulses evoked graded potentials in the muscle cells inervated by these neurons (Fig. 7). Intracellularly recorded muscle potentials did not show a threshold; rather, they were continuously graded up to a maximum value. The maximal response 


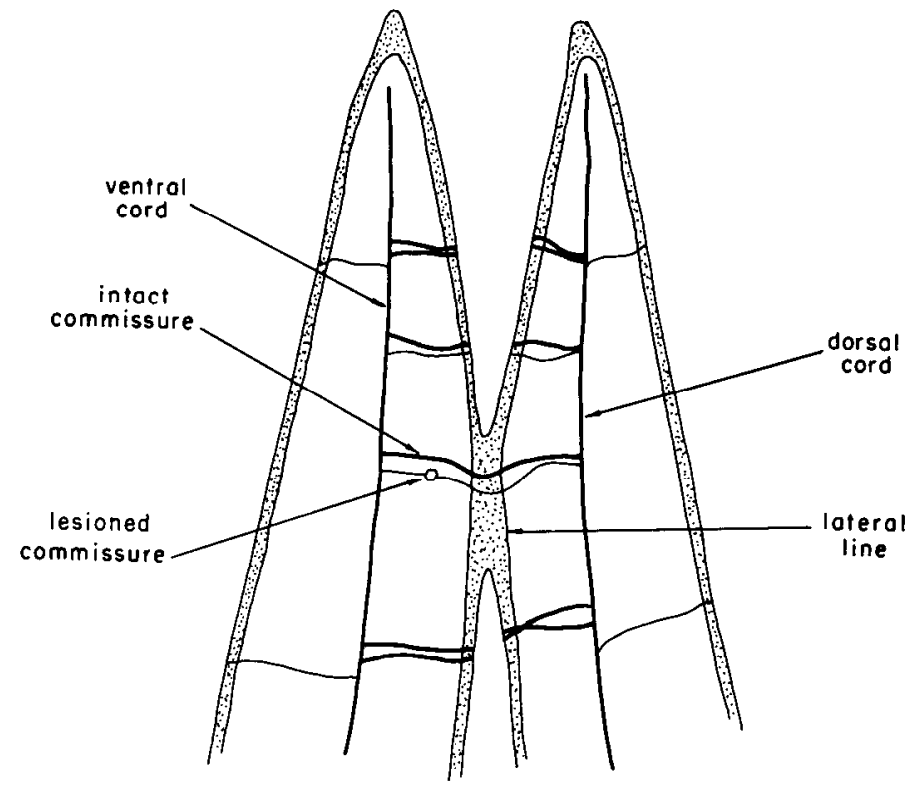

Figure 2. Diagram of preparation used to stimulate DE1 motoneurons. The animal has been cut along the left lateral line and pinned out with the cuticle side down. The right lateral line has been cut, leaving a narrow bridge of tissue connecting the dorsal and ventral halves of the animal. A pair of commissures traverse the bridge. One member of the pair has been severed with an insect pin so that the dorsal and ventral nerve cords remain connected by a single commissure. Muscle activity evoked by stimulating the intact neuron in one nerve cord can be recorded intracellularly in the muscle cells innervated at the other cord. In the diagram, the muscle cells have been omitted.

varied from cell to cell but seldom exceeded $30 \mathrm{mV}$. These results indicate that a single excitatory motoneuron can produce a continuously graded electrical response in the muscle cells it innervates. Hyperpolarizing responses to stimulation of inhibitory motoneurons are also graded (results not shown).

The morphological features revealed by analyses of selected serial sections in the electron microscope had previously been used to identify for each motoneuron the input region, which receives synapses from other neurons, and the output region, where the neuron synapses onto muscle (Stretton et al., 1978). All of the experiments described so far have concentrated on stimulating the motoneuron's input region while recording from muscle cells along its output region. Complementary experiments were carried out in which the output region of the cells was stimulated while recording from muscle cells along the input region. Even when signal averaging was used to detect low amplitude responses, activation of the output region failed to produce detectable responses in muscle cells along the input region.

A total of 94 determinations of function were performed on 21 of the 22 motoneurons with commissures in the first three segments (Table I). (The neuron with the single extra righthand commissure in the first segment was not investigated.) In 63 experiments the intact neuron was rigorously identified by histological means after completion of the physiological experiment (Fig. 8, Table I).

\section{Discussion}

Physiological properties of identified neurons. The motoneurons of Ascaris are organized in a repeating pattern within the ventral and dorsal nerve cords (Stretton et al., 1978). Each repeating unit or segment contains a set of 11 motoneurons; on the basis of their morphology, these have been divided into seven different classes. Members of three classes are present once in each repeat, whereas four classes are represented twice $(3+(4 \times 2)=11)$. The results presented in this paper have determined the physiological sign of neuromuscular transmission of representatives of five of the seven types of motoneurons - three (DE1, DE2, and DE3) are excitatory; two (DI and VI) are inhibitory (Fig. 9).

Each of 21 different neurons with commissures that we investigated in the first three segments (i.e., all of those present except for the extra single right-hand commissure in the first segment) had been assigned to one of five classes based on anatomical criteria. In the electrophysiological experiments reported in this paper, different members of the same class of neuron evoke similar responses in the musculature, so that there is an excellent correlation between structure and function in the set of neurons we have studied. In the case of the first pair of commissures in the first segment, they had been classified as a DEl/VI pair on the basis of the morphology and relative position of their input and output processes (Stretton et al., 1978), even though their commissures are reflected to the left. The physiological experiments confirmed that this assignment was correct.

Further experiments have shown that these functional analogies extend to the biochemical level as well; commissures of the DE1, DE2, and DE3 motoneurons contain choline acetyltransferase, the enzyme which synthesizes acetylcholine (ACh), whereas those of DI and VI do not (C. D. Johnson and A. O. W. Stretton, submitted for publication). This suggests that $\mathrm{ACh}$ is the neurotransmitter released by the DE motoneurons. The neurotransmitter released by inhibitory motoneurons has not yet been as well defined, although it has been suggested on the basis of pharmacological experiments that $\gamma$-aminobutyric acid may be the inhibitory transmitter (del Castillo el al., 1964).

The physiological results are completely congruent with the anatomical categorization of the dorsal or ventral process of each commissural neuron as either an input or a neuromuscular output zone (Stretton et al., 1978). However, the fact that evoked responses are recorded only in the anatomically defined output region must be interpreted cautiously. First, unless complete serial section electron microscopy is carried out, our anatomical data have only shown that any neuromuscular synapses made by the morphologically defined dendritic zone are rare, not that they do not exist. Second, although no evoked signals are detectable in muscle cells in the input zone of a neuron when its output process is stimulated, this could be due to failure of propagation of the antidromic signal into the commissure or into its dendritic process. Intracellular recording from the commissure and the dorsal and ventral processes of each neuron will be necessary before a completely satisfactory interpretation of these particular results can be made.

Since the remaining two classes of motoneurons, types V-1 and $\mathrm{V}-2$, are confined to the ventral nerve cord and do not have commissures, the direct physiological investigation of their function by the methods used for the commissural motoneurons is not possible. We surmise, on the basis of structural analogies with DE neurons, that both of these neuron types are ventral excitatory motoneurons. First, the V-1 and V-2 neurons extend a ventral process which makes synapses to ventral muscle cells in only one direction, either anteriorly (V-1) or posteriorly ( $\mathrm{V}$ 2), relative to their cell bodies (Fig. 9). The dorsal excitors extend a dorsal process in only one direction from the commissure and cell body, either anteriorly (DE1 and DE3) or posteriorly (DE2). In contrast, both types of inhibitory motoneurons (DI and VI) extend processes both anteriorly and posteriorly in both nerve cords. (Structurally, the DI neurons seem to be the dorsal equivalent of the VI neurons.) In addition, the locations of the DE and V-motoneurons within the two nerve cords are similar. In transverse sections of the nerve cords, the profiles of motoneurons (Fig. 10) fall into groups; thus, in the 

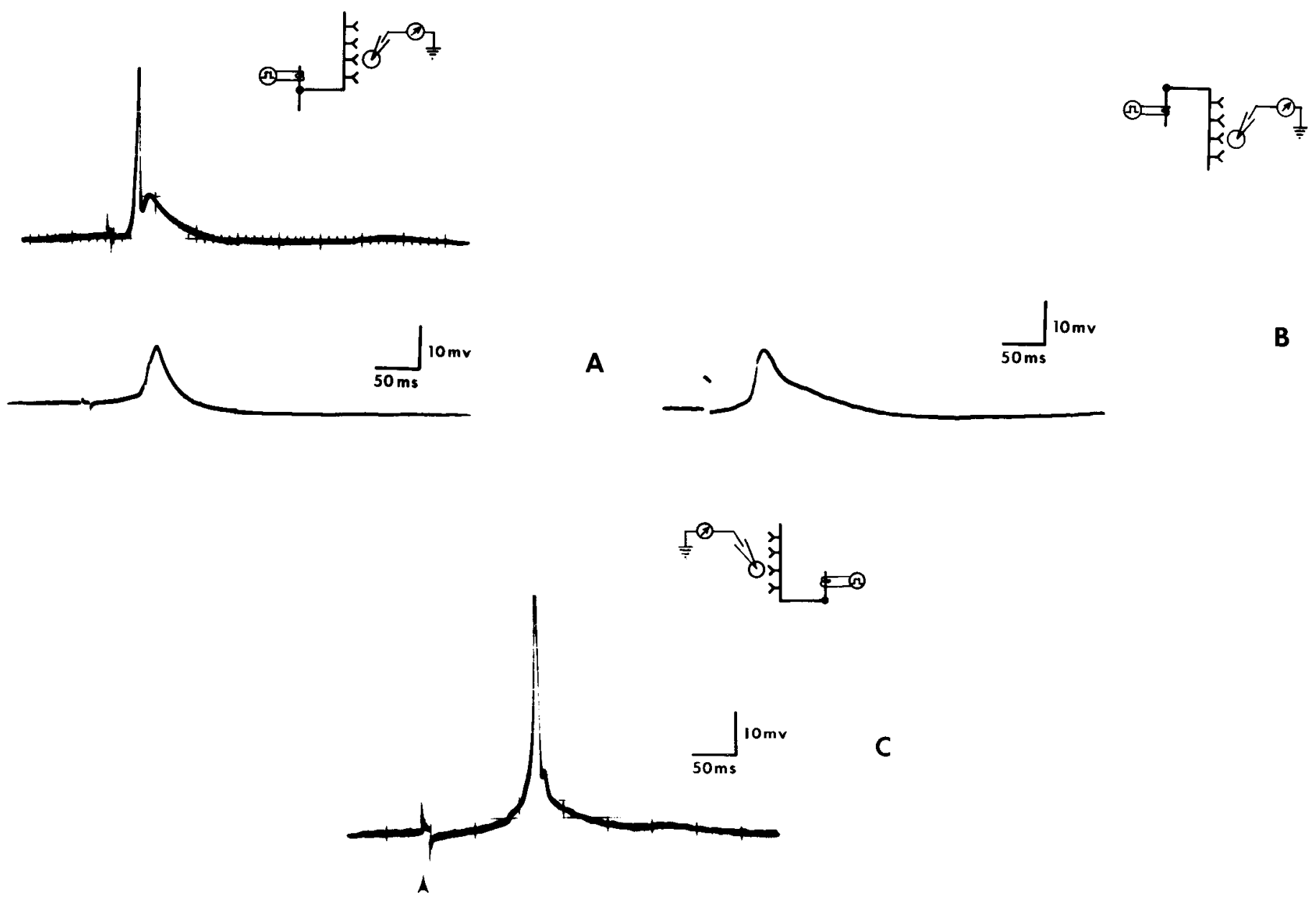

Figure 3. Physiological responses of the DE motoneurons. The inset in the upper right of each panel shows the shape of the DE1 (A), DE2 $(B)$, and DE3 $(C)$ motoneurons, the neuromuscular output region of the cell (marked by Y's), and the position of the stimulating (circle with square wave) and recording (circle with arrow) electrodes. DE neurons evoke depolarizing responses in the dorsal musculature. In more lateral muscles, the responses are usually slow depolarizations (e.g., $B$, and bottom record of $A$ ), whereas in more medial muscles, slow depolarizations with superimposed spikes occur (e.g., $C$, and top record of $A$ ). Examples of each type of response are shown. Scale: vertical, $10 \mathrm{mV}$; horizontal, $50 \mathrm{msec}$.

dorsal cord the inhibitory neurons form a group on one side of the cord, and the dorsal excitatory neurons form a group on the other side. An analogous arrangement of motoneurons exists in the ventral cord where the inhibitory neurons are grouped on one side, and the V-1 and V-2 neurons are on the other side. Thus, on these structural criteria the V-1 and V-2 neurons are equivalent to the dorsal excitors.

A more convincing argument can be made by considering the synaptic connections made by these neurons. The DE1, DE2, and DE3 neurons receive input from interneurons in the ventral cord. In the dorsal cord, besides making neuromuscular synapses, they form the sole source of input to the dendrites of ventral inhibitory neurons (see Walrond and Stretton, 1985a) and make synapses onto the neuromuscular output regions of the dorsal inhibitors (see Walrond and Stretton 1985b). Similarly, in the ventral cord the V-1 and V-2 neurons receive their input from interneurons, make ventral neuromuscular synapses, provide the sole input to the dendrites of the dorsal inhibitors, and make synapses with the ventral inhibitory output regions (J. E. Donmoyer, P. A. Desnoyers, and A. O. W. Stretton, unpublished observations). Taken together, these anatomical data show that the shape, position, and synaptic interactions of V-1 and V-2 neurons are analogous to those of the dorsal excitory motoneurons; therefore, we suppose that both V-1 and V-2 neurons are excitatory. Direct physiological evidence is needed to establish this conclusion firmly.

Graded neuromuscular transmission. By varying the parameters of stimulation, we observed that the response recorded in muscle cells did not show an all-or-none threshold but was continuously graded with the strength of the stimulus. This suggests that neither the neurons nor the muscle cells propagate all-or-nothing action potentials. In the experiments described in this paper, electrical activity of motoneurons has been inferred from recording their effects upon muscle cells, rather than by recording from the neurons. Direct intracellular recordings from the neurons have shown that, indeed, the neurons do not propagate action potentials, and that neuromuscular transmission is graded (Davis and Stretton, 1982). The relationship between neuronal signals and muscle responses is not linear; the input-output properties of the synapse, amplification produced by active channels in the muscle cell (del Castillo et al., 1967), and the presence of electrical synapses between muscle cells (del Castillo et al., 1963) will each affect this relationship, but the relative contribution of each of these factors has yet to be determined.

Complex responses. Complex responses in muscle cells were often seen: in the case of excitors, the initial evoked depolarization was often followed by a hyperpolarization, and for inhibitors, the initial hyperpolarization was often followed by a 

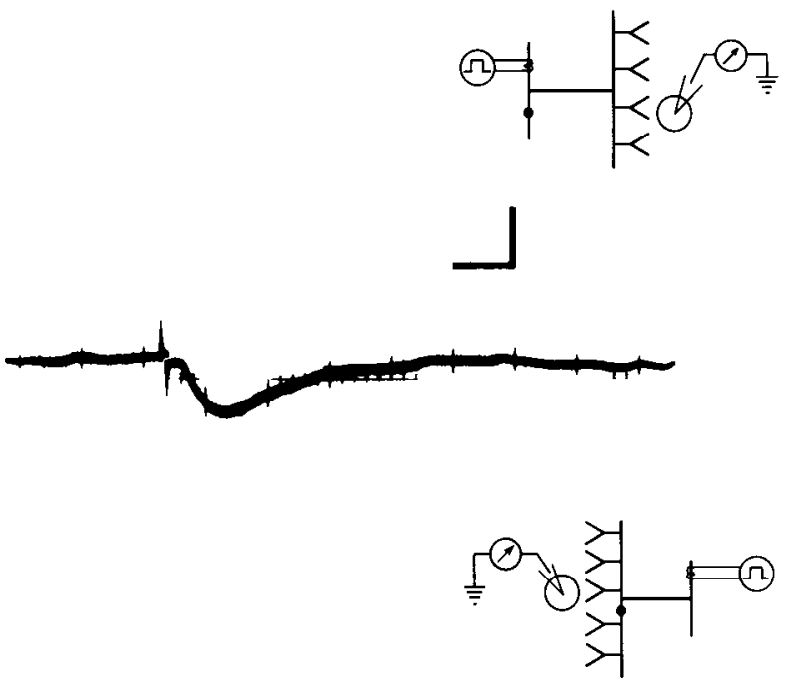

B

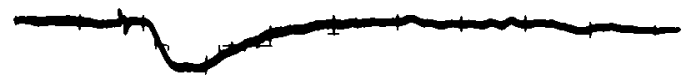

Figure 4. Physiological responses of the DI and VI motoneurons. The diagram shows the shape of the DI $(A)$ and VI $(B)$ motoneurons. Symbols are as in Figure 3. Activation of DI motoneurons evokes hyperpolarizing responses from the dorsal musculature. Activation of a VI neuron evokes hyperpolarizing responses in ventral muscle cells. Scale: vertical, $5 \mathrm{mV}$; horizontal, $100 \mathrm{msec}$.

a

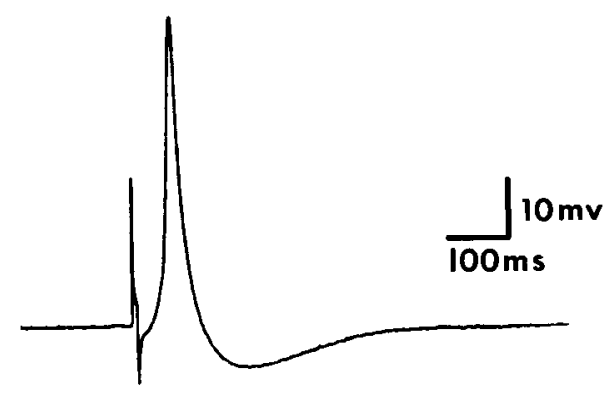

b

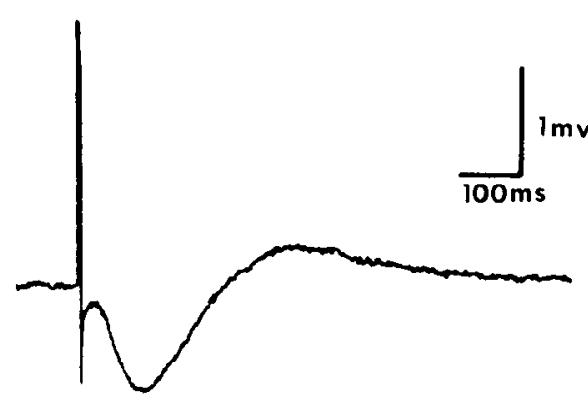

Figure 5. Biphasic responses to stimulation of $\mathrm{E}$ and I motoneurons. $a$, Activation of a DE1 motoneuron evokes a depolarization followed by a hyperpolarizing action. $b$, Activation of a VI motoneuron evokes a hyperpolarization followed by a depolarization.

depolarization. The mechanisms generating the secondary responses are not yet understood, but based on our anatomical findings, we would suggest that the most probable explanation is that neuron-neuron interactions in the dorsal cord are responsible. We have shown by electron microscopy that there

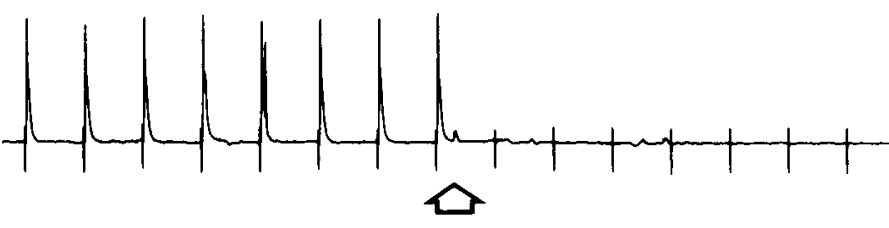

Figure 6. Evoked responses require intact commissures. The record shows the dorsal muscle response evoked by the repetitive ventral activation of a DE2 motoneuron. 'The responses follow the stimulus one for one until the commissure is cut (arrow), whereupon the response is obliterated.

\section{TABLE I}

Muscle responses evoked by individual motoneurons

The first column lists the names of commissural motoneurons in the first three segments. The first letter of the name designates whether the neuron has its neuromuscular output to dorsal (D) or ventral (V) muscles; the second letter designates whether its neuromuscular output is excitatory (E) or inhibitory (I); and the number following the second letter differentiates the three types of dorsal excitors. Neurons present in two copies per segment are labeled with superscripts ( 1 or 2$)$ and indicate the anterior or posterior copy, respectively. The second column gives the number of experiments performed on each neuron, and the response recorded ( $\mathrm{F}$ or $\mathrm{I}$ ) is given in the third and fourth columns. The fifth column shows the number of experiments in which the commissure was cut while recording the evoked response. The sixth column lists the number of preparations in which the identification of intact and cut neurons was verified by histological procedures.

\begin{tabular}{|c|c|c|c|c|c|}
\hline \multirow{2}{*}{ Neuron } & \multirow{2}{*}{$\begin{array}{c}\text { No. of } \\
\text { Experiments }\end{array}$} & \multicolumn{2}{|c|}{$\begin{array}{c}\text { Re- } \\
\text { sponse }\end{array}$} & \multirow[t]{2}{*}{ No. Cut } & \multirow{2}{*}{$\begin{array}{l}\text { Histologica } \\
\text { Verification }\end{array}$} \\
\hline & & $\mathrm{E}$ & $\mathrm{I}$ & & \\
\hline \multicolumn{6}{|l|}{ Segment I } \\
\hline $\mathrm{DE} 1^{1}$ & 3 & 3 & 0 & 0 & 0 \\
\hline $\mathrm{VI}^{1}$ & 2 & 0 & 2 & 2 & 1 \\
\hline $\mathrm{DE} 1^{2}$ & 5 & 5 & 0 & 3 & 0 \\
\hline $\mathrm{VI}^{2}$ & 3 & 0 & 3 & 0 & 0 \\
\hline DE2 & 2 & 2 & 0 & 1 & 0 \\
\hline DI & 4 & 0 & 4 & 2 & 0 \\
\hline DE3 & 3 & 3 & 0 & 2 & 0 \\
\hline \multicolumn{6}{|c|}{ Segment II } \\
\hline $\mathrm{DE}^{1}$ & 4 & 4 & 0 & 2 & 4 \\
\hline $\mathrm{VI}^{1}$ & 6 & 0 & 6 & 1 & 5 \\
\hline$D E 1^{2}$ & 5 & 5 & 0 & 4 & 5 \\
\hline $\mathrm{VI}^{2}$ & 7 & 0 & 7 & 1 & 7 \\
\hline DE2 & 7 & 7 & 0 & 2 & 5 \\
\hline DI & 5 & 0 & 5 & 0 & 5 \\
\hline DE3 & 5 & 5 & 0 & 4 & 5 \\
\hline \multicolumn{6}{|c|}{ Segment III } \\
\hline $\mathrm{DE} 1^{1}$ & 3 & 3 & 0 & 1 & 3 \\
\hline $\mathrm{VI}^{1}$ & 5 & 0 & 5 & 3 & 5 \\
\hline $\mathrm{DE} 1^{2}$ & 5 & 5 & 0 & 1 & 5 \\
\hline $\mathrm{VI}^{2}$ & 5 & 0 & 5 & 0 & 5 \\
\hline DE2 & 5 & 5 & 0 & 0 & 2 \\
\hline DI & 6 & 0 & 6 & 2 & 3 \\
\hline DE3 & 4 & 4 & 0 & 1 & 4 \\
\hline
\end{tabular}

are numerous reciprocal synapses between DE and DI neurons in the dorsal cord (P. A. Desnoyers, J. E. Donmoyer, and A. O. W. Stretton, unpublished observation). Thus, in each case there are two major pathways between motoneurons and muscle. One is monosynaptic, the other disynaptic: DE motoneurons synapse onto muscle and onto DI motoneurons which also synapse onto muscle (and vice versa for the DI motoneurons). Such a local circuit interaction could account for the hyperpolarization following a DE-evoked depolarizing response. In the case of the 


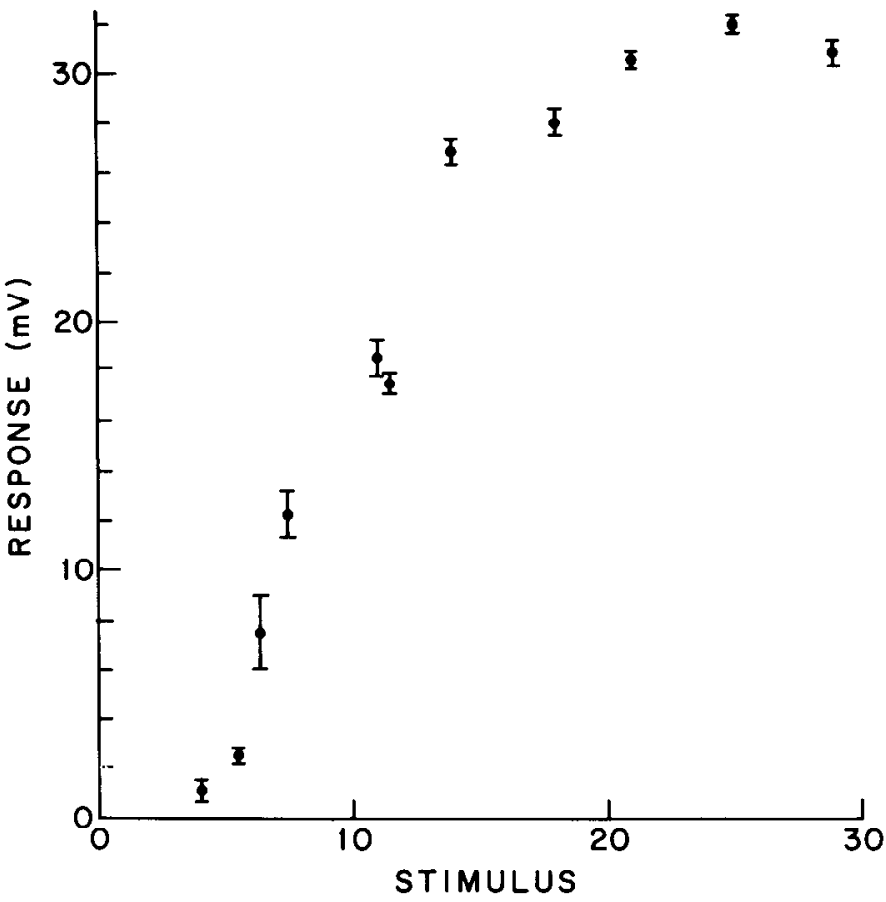

Figure 7. Graded responses in muscle cells. A DE1 motoneuron was stimulated ventrally with a suction electrode while recording intracellularly from a muscle cell innervated by this neuron. For each stimulus strength, 20 responses were averaged. Results show standard error of the mean. Stimulus strength is indicated in arbitrary units.

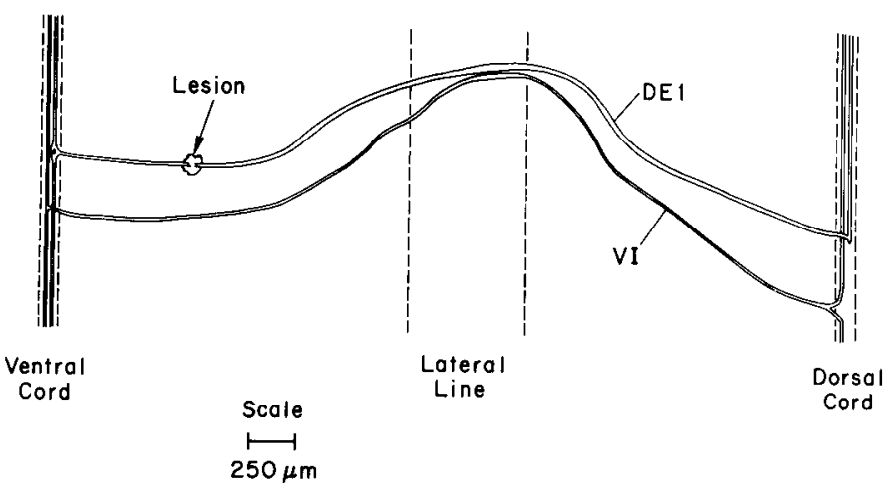

Figure 8. Reconstruction from serial $10-\mu \mathrm{m}$ sections of a DE1/VI pair of commissures in which the DE1 commissure had been lesioned. The individual neurons were reconstructed by tracing their profiles in the nerve cords: the DE1 neuron has a cell body at the origin of its commissure and sends both anterior and posterior processes in the ventral cord, but only an anterior process in the dorsal cord; the VI neuron sends processes both anteriorly and posteriorly in both nerve cords and often branches before it enters the dorsal cord. The DE1 commissure is usually thicker than the VI commissure. The diameter of the neuron processes is not to scale: the diameter of the DE1 commissure was $\sim 16 \mu \mathrm{m}$, and that of the VI commissure was $\sim 8 \mu \mathrm{m}$.

DI-evoked muscle hyperpolarization, the subsequent depolarization could be due to excitatory transmitter release caused by rebound following hyperpolarization of the DE neuron. Clearly, until the electrophysiological properties of these neuron-neuron synapses are fully described, these explanations are speculative, and alternative mechanisms, e.g., the electrical properties of the muscle cells, or multiaction synapses (Kandel et al., 1967; Getting, 1981), remain a distinct possibility.

Correlations with Caenorhabditis elegans neurons. There is a remarkable morphological similarity between the motoneurons of Caenorhabditis elegans (White et al., 1976) and Ascaris
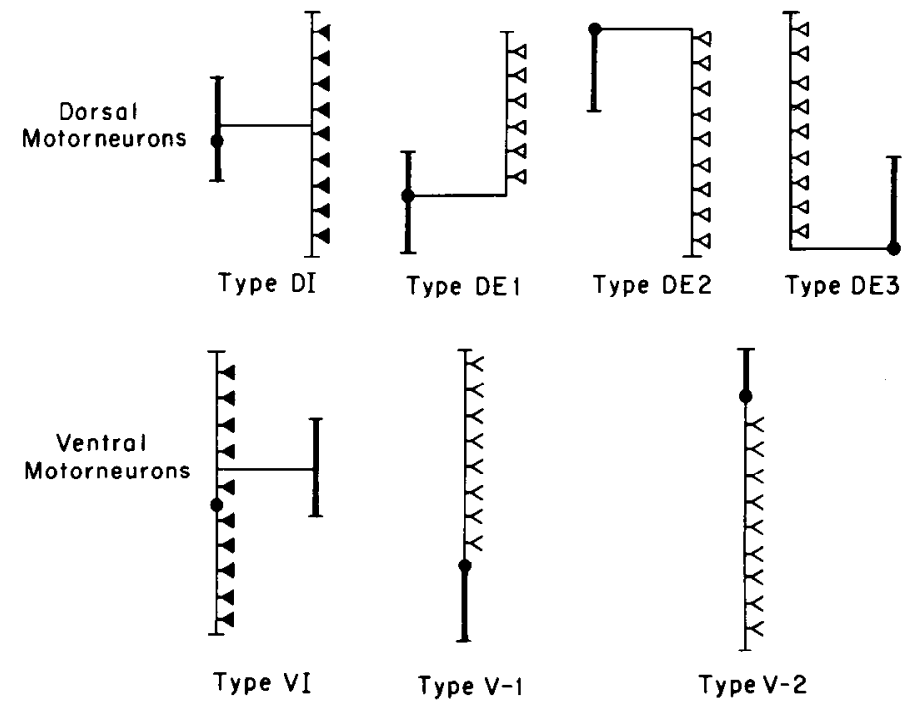

Figure 9. The location of the dendritic and neuromuscular output regions for each of the seven neuronal types. The $Y^{\prime}$ 's and triangles represent the neuromuscular output regions. Neurons in the top row have dorsal neuromuscular output regions, and neurons in the bottom row have ventral neuromuscular output regions. The open triangles represent excitatory output to muscle, and the solid triangles represent inhibitory output. The Y's on the V-1 and V-2 neurons indicate that the sign of the output to muscle has not been determined physiologically. The thickened lines represent the input or dendritic regions for each neuron type.
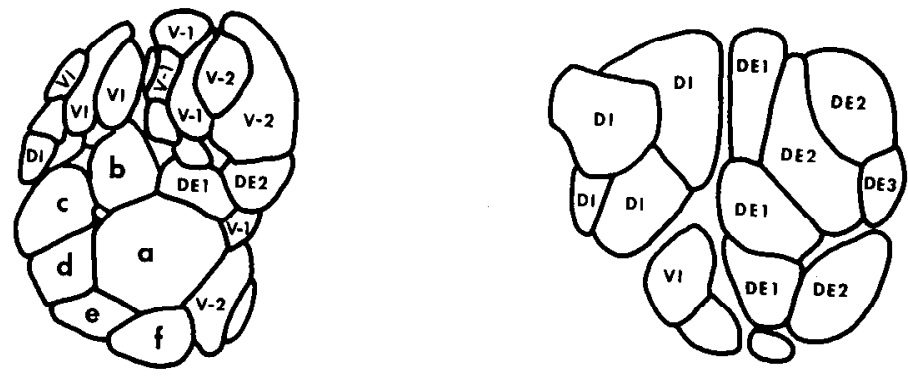

V

D

Figure 10. Tracings of transverse sections of neurons in the ventral $(V)$ and dorsal $(D)$ nerve cords. The neuromusclar junctions in each case are made at the top of the perimeter. $a$ to $f$ are profiles of the large ventral cord interneurons. Profiles of motoneurons are labeled according to their type. Small unlabeled profiles between excitors and inhibitors in the ventral cord are small interneurons.

(Stretton et al., 1978). The shapes and locations of the neuromuscular output regions of individual cells allow each motoneuron type in Ascaris to be given a precise equivalent in Caenorhabditis (Table II). Neuron types DAS, DB, and DA in Caenorhabditis correspond to types DE1, DE2, and DE3, respectively, and therefore are probably excitatory to the dorsal muscles. The inhibitory motoneurons DI and VI are equivalent to the Caenorhabditis DD and VD neurons, indicating that they may be the inhibitory motoneurons. Finally, VA and VB neurons in Caenorhabditis correspond to the V-1 and V-2 of Ascaris neurons which are probably excitatory.

In C. elegans, the DE2, DE3, and DI motoneurons result from embryonic cell lineages (Sulston et al., 1983). The DE1, 
TABLE II

Structurally equivalent motoneurons in Ascaris and Caenorhabditis ${ }^{a}$

\begin{tabular}{cl}
\hline A. lumbricoides & C. elegans $^{a}$ \\
\hline DE1 & DAS \\
DE2 & DB \\
DE3 & DA \\
DI & DD \\
VI & VD \\
V-1 & VA \\
V-2 & VB \\
\hline
\end{tabular}

${ }^{a}$ For C. elegans nomenclature see White et al. (1976).

VI, V-1, and V-2 motoneurons are produced postembryonically (Sulston, 1976). In first stage larvae, neuron types DE3 and DE2 innervate the dorsal musculature and synapse onto the dorsal process of the DI neuron which (in this stage only) innervates the ventral musculature (White et al., 1978). (This neuron later rewires, interchanging axonal and dendritic regions during the second larval stage.) Assuming that the neurotransmitters and postsynaptic receptors are identical in the larva and the adult, it seems, based on Ascaris motoneuron physiology, that movement of the first stage larva is controlled by two dorsal excitors and one ventral inhibitor. Both dorsal inhibitory and ventral excitatory motoneurons are absent.

Studies in nematode neurobiology are particularly attractive, since information from studies on one species may be applicable to the other. Considering the evolutionary distance between Ascaris and C. elegans, the structural resemblance is remarkable and may imply a basic conservatism in the organization of the nematode nervous system. Although $C$. elegans has proved advantageous in anatomical, developmental, and genetic studies, its small size has so far prevented electrophysiological investigations. Conversely, the large size of Ascaris facilitates physiological investigations but hampers developmental and genetic studies. Combining information gained from these diverse approaches to these two species in which neuronal structure is so similar may provide insights into the interrelationships of the genetic, developmental, and physiological basis of behavior.

\section{References}

Bullock, T. H., and G. A. Horridge (1965) Structure and Function in the Nervous System of Invertebrates, p. 606, W. H. Freeman and Co., San Francisco.

Davis, R. E., and A. O. W. Stretton (1981) Intracellular recordings from identified motorneurons in the nematode Ascaris. Soc. Neurosci. Abstr. 7: 745 .

Davis, R. E., and A. O. W. Stretton (1982) Motorneuron membrane constants and signaling properties in the nematode Ascaris. Soc. Neurosci. Abstr. 8: 685 .

Davis, R. E., and A. O. W. Stretton (1983) Motorneuron signaling properties and motorneuron-motorneuron synaptic interactions in the nematode Ascaris. Soc. Neurosci. Abstr. 9: 301.

de Bell, J. T., J. del Castillo, and V. Sanchez (1963) Electrophysiology of the somatic muscle cells of Ascaris lumbricoides. J. Cell. Comp. Physiol. 62: 159-177. del Castillo, J., W. C. de Mello, and T. Morales (1963) The physiological role of acetylcholine in the neuromuscular system of Ascaris lumbricoides. Arch. Int. Physiol. Biochim. 71: 741-757.

del Castillo, J., W. C. de Mello, and T. Morales (1964) Inhibitory action of $\gamma$-aminobutyric acid (GABA) on Ascaris muscle. Experientia 20: $141-143$.

del Castillo, J., W. C. de Mello, and T. Morales (1967) Initiation of action potentials in the somatic musculature of Ascaris lumbricoides. J. Exp. Biol. 46: 263-279.

Getting, P. A. (1981) Mechanisms of pattern generation underlying swimming in Tritonia. I. Neuronal network formed by monosynaptic connections. J. Neurophysiol. 46: 65-79.

Goldschmidt, R. (1908) Das Nervensystem von Ascaris lumbridoides und megalocephala. I. Z. Wiss. Zool. 90: 73-136.

Goldschmidt, R. (1909) Das Nervensystem von Ascaris lumbricoides und megalocephala. II. Z. Wiss. Zool. 92: 306-357.

Hesse, R. (1892) Uber das Nervensystem von Ascaris megalacephala. Z. Wiss. Zool. 54: 548-568.

Jarman, M. (1959) Electrical activity in the muscle cells of Ascaris lumbricoides. Nature 184: 1244.

Kandel, E. R., W. T. Frazier, R. Waziri, and R. E. Coggeshall (1967) Direct and common connections among identified neurons in Aplysia. J. Neurophysiol. 30: 1352-1376.

Rosenbluth, J. (1965) Ultrastructure of somatic muscle cclls in Ascaris lumbricoides. J. Cell Biol. 26: 579-591.

Stretton, A. O. W. (1976) Anatomy and development of the somatic musculature of the nematode Ascaris. J. Exp. Biol. 64: 773-788.

Stretton, A. O. W., R. M. Fishpool, E. Southgate, J. E. Donmoyer, J. P. Walrond, J. E. R. Moses, and I. S. Kass (1978) Structure and physiological activity of the motorneurons of the nematode Ascaris. Proc. Natl. Acad. Sci. U. S. A. 75; 3493-3497.

Sulston, J. E. (1976) Post-embryonic development in the ventral cord of Caenorhabditis elegans. Philos. Trans. R. Soc. Lond. (Biol.) 275: 287-297.

Sulston, J. F., F. Schierenberg, I. G. White, and J. N. Thomson (1983) The embryonic cell lineage of the nematode Caenorhabditis elegans. Dev. Biol. 100: 64-119.

Walrond, J. P. (1979) Actions and interactions in Ascaris: A plausible mechanism for neural control of locomotion in nematodes. Ph.D. thesis, University of Wisconsin, Madison.

Walrond, J. P., and A. O. W. Stretton (1985a) Reciprocal inhibition in the motor nervous system of the nematode Ascaris: Direct control of ventral inhibitory motoneurons by dorsal excitatory motoneurons. J. Neurosci. 5: 9-15.

Walrond, J. P., and A. O. W. Stretton (1985b) Excitatory and inhibitory activity in the dorsal musculature of the nematode Ascaris evoked by single dorsal excitatory motoneurons. J. Neurosci. 5: 16-22.

Walrond, J. P., I. S. Kass, J. E. Donmoyer, J. E. R. Moses, and A. O. W. Stretton (1977) Flectrophysiology and anatomy of identified neurons of Ascaris. Soc. Neurosci. Abstr. 3: 190.

Walrond, J. P., J. E. Donmoyer, P. A. Desnoyers, and A. O. W. Stretton (1978) Synaptic interactions between motorneurons of Ascaris. Soc. Neurosci. Abstr. 4: 209.

Weisblat, D. A., and R. L. Russell (1976) Propagation of electrical activity in the nerve cord and muscle syncytium of the nematode Ascaris lumbricoides. J. Comp. Physiol. A 107: 293-307.

White, J. G., E. Southgate, J. N. Thomson, and S. Brenner (1976) The structure of the ventral nerve cord of Caenorhabditis elegans. Philos. Trans. R. Soc. London (Biol.) 275: 327-348.

White, J. G., D. G. Albertson, and M. A. R. Anness (1978) Connectivity changes in a class of motorneurons during the development of a nematode. Nature 271: 764-766. 